\title{
BINOL-derived bifunctional sulfide catalysts for asymmetric synthesis of 3,3-disubstituted phthalides via bromolactonization
}

Received 00th January 20xx, Accepted 00th January 20xx

\author{
Megumi Okada, ${ }^{a}$ Kazuma Kaneko, ${ }^{\mathrm{b}}$ Masahiro Yamanaka ${ }^{\mathrm{b}}$ and Seiji Shirakawa*a
}

DOI: $10.1039 / \times 0 \times x 00000 x$

\begin{abstract}
Efficient enantioselective synthesis of 3,3-disubstituted phthalides possessing a chiral quaternary carbon center was achieved via catalytic asymmetric bromolactonization that utilized BINOLderived bifunctional sulfide catalysts. Transformations of the bromo group in optically active phthalide products were also performed to demonstrate the utility of this novel synthetic protocol.
\end{abstract}

The phthalide structure appears in many important natural products and biologically active compounds, which is why phthalides are recognized as some of the most important lactones (Scheme 1). ${ }^{1}$ There have been numerous attempts to build this structural motif, which includes the asymmetric synthesis of chiral phthalides. ${ }^{2}$ A catalytic asymmetric synthesis of 3,3-disubstituted phthalides possessing a chiral quaternary carbon center ranks among the most important challenges in modern organic synthesis. Catalytic enantioselective fluoroand chlorolactonizations of substrate $\mathbf{1}$ were recently developed as effective methods to produce 3,3-disubstituted phthalides. 3,4 Unfortunately, a catalytic asymmetric method for the bromolactonization of $\mathbf{1}$ has remained elusive, despite great expectations for the synthetic utility of the resultant bromosubstituted phthalides $\mathbf{2}$ for use in further transformations. ${ }^{5}$ In the course of our recent study into the development of chiral bifunctional sulfide catalysts (S)-3 for bromolactonization to produce chiral 3,4-dihydroisocoumarins, ${ }^{6}$ we became interested in the construction of 3,3-disubstituted chiral phthalides via the asymmetric bromolactonization of $\mathbf{1}$ with our sulfide catalysts (Scheme 1). ${ }^{7,8}$ Our previously developed bifunctional sulfide (S)-3 possesses a urea moiety, but has shown poor selectivity for the present reaction. However, redesigned sulfide catalysts (S)-4 bearing a hydroxy group improved the enantioselectivity. Herein, we report our efforts

\footnotetext{
a. Department of Environmental Science, Graduate School of Fisheries and Environmental Sciences, Nagasaki University, 1-14 Bunkyo-machi, Nagasaki 8528521, Japan. E-mail: seijishirakawa@nagasaki-u.ac.jp

b. Department of Chemistry and Research Center for Smart Molecules, Faculty of Science, Rikkyo University, 3-34-1, Nishi-lkebukuro, Toshima-ku, Tokyo 171-8501, Japan

† Footnotes relating to the title and/or authors should appear here.

Electronic Supplementary Information (ESI) available: [details of any supplementary information available should be included here]. See DOI: 10.1039/x0xx00000x
}

to enantioselectively synthesize 3,3-disubstituted phthalides 2 using chiral bifunctional sulfide catalysts. Transformations of the bromo group in optically active phthalide products $\mathbf{2}$ were also performed to demonstrate the potential utility of the present synthetic protocol.
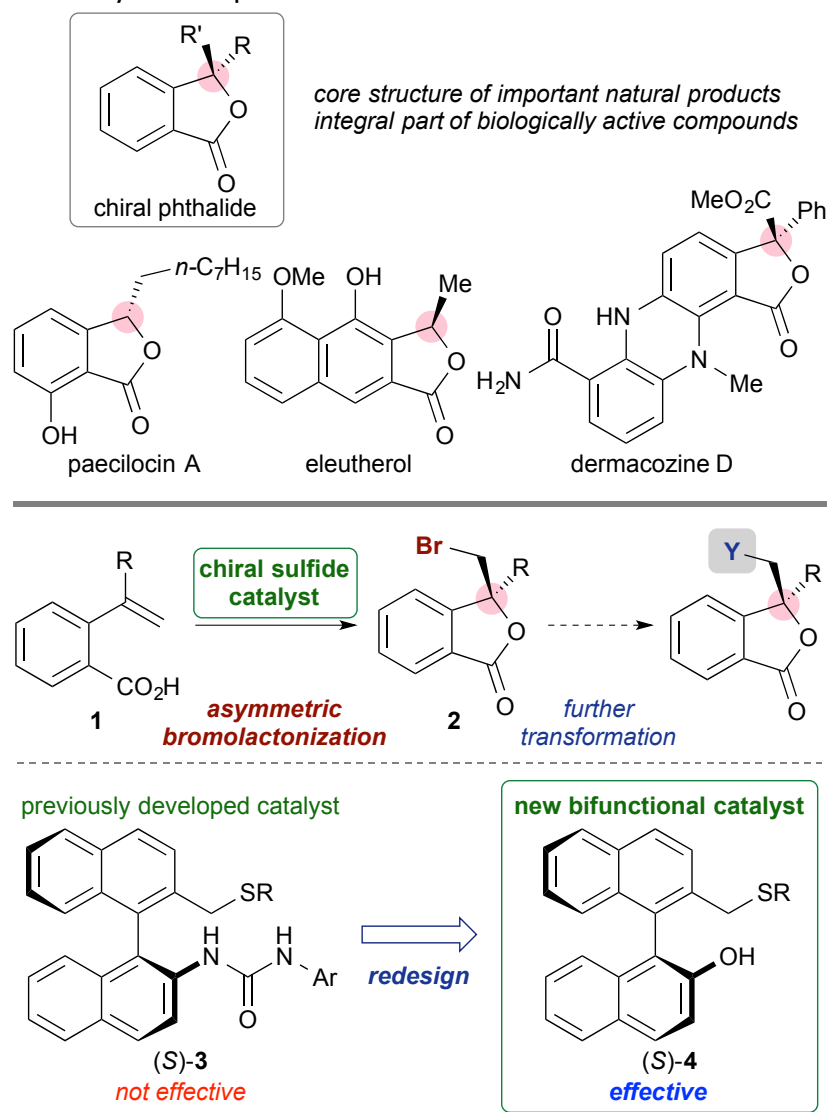

Scheme 1 Important chiral phthalides and our synthetic approach.

Our initial aim was to find an effective catalyst for the asymmetric bromolactonization of $\mathbf{1}$ (Table 1). An attempted reaction of 1a with $\mathrm{N}$-bromophthalimide (NBP) in toluene$\mathrm{CH}_{2} \mathrm{Cl}_{2}$ under the influence of a urea-type bifunctional sulfide catalyst (S)-3a, ${ }^{9}$ which is an effective catalyst for different enantioselective bromolactonizations, ${ }^{6}$ at $-78{ }^{\circ} \mathrm{C}$ for $24 \mathrm{~h}$ 
afforded the target phthalide product $2 \mathrm{a}$ in a high yield, but with poor enantioselectivity (53:47 er, entry 1 ). This result prompted us to examine different types of chiral sulfide catalysts. To our delight, the reaction with a relatively simple bifunctional sulfide (S)-4a possessing a hydroxy group gave product 2 a with good enantioselectivity (88:12 er, entry 2 ). To clarify the role of the hydroxy group in bifunctional catalysts $(S)-4$, we also examined a reaction with hydroxy-protected mono-functional catalyst $(S)$ 5a. As expected, the reaction with mono-functional catalyst $(S)$ $5 \mathbf{a}$ produced $\mathbf{2} \mathbf{a}$ in a low level of enantioselectivity with opposite configuration of the major isomer (43:57 er, entry 3 ). These results suggested that the bifunctional design of sulfide catalysts (S)-4 bearing a hydroxy group is essential in order to obtain a high level of enantioselectivity. Encouraged by these results, a fine-tuning of the sulfide moiety on the catalyst (S)-4 was performed to improve the enantioselectivity. The higher enantioselectivities of product $2 \mathrm{a}$ were observed in the reaction using catalysts $(S)-\mathbf{4 b}(\mathrm{R}=i$ - $\mathrm{Pr}, 94: 6$ er, entry 4$)$ and $\mathbf{4 c}(\mathrm{R}=t-\mathrm{Bu}$, 93:7 er, entry 5), which possess a more bulky alkyl group on the sulfur. Although we generally performed the reaction for $24 \mathrm{~h}$, the actual reaction was almost completed within $6 \mathrm{~h}$ (entry 6). The introduction of substituents at the 3-position of a binaphthyl unit on the catalyst did not improve the enantioselectivity (catalysts (S)-6a-c, entries 7-9). It should be noted that the present reaction efficiently proceeded even without a catalyst (entry 10).

Table 1 Catalyst optimization ${ }^{a}$<smiles>C=C(c1ccccc1)c1ccccc1C(=O)O</smiles>

1a<smiles>O=C1c2ccccc2C(=O)N1Br</smiles>

NBP

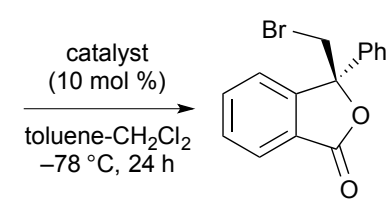

2a<smiles>CCCCc1ccc2ccccc2c1-c1c(NC(=O)NF)ccc2ccccc12</smiles>

(S)-3a<smiles>Oc1ccc2ccccc2c1-c1c(CS)ccc2ccccc12</smiles>

(S)-4a: $\mathrm{R}=n-\mathrm{Bu}$ (S)-4 $\quad$ (S)-4c: $R=t-B u$<smiles>COc1ccc2ccccc2c1-c1c(C[Sn]C(C)(C)C)ccc2ccccc12</smiles>

(S) $-5 \mathbf{a}$

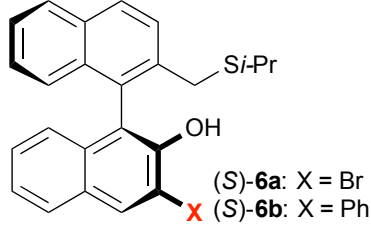

$\begin{array}{ll}\text { (S)-6 } & \text { (S)-6c: } X=3,5-\mathrm{Ph}_{2}-\mathrm{C}_{6} \mathrm{H}_{3}\end{array}$

\begin{tabular}{llll}
\hline Entry & Catalyst & Yield $^{b}(\%)$ & $\mathrm{er}^{c}$ \\
\hline 1 & $(S)-3 \mathbf{a}$ & 99 & $53: 47$ \\
2 & $(S)-4 \mathbf{a}$ & 92 & $88: 12$ \\
3 & $(S)-5 a$ & 97 & $43: 57$ \\
4 & $(S)-4 b$ & 98 & $94: 6$ \\
5 & $(S)-4 \mathbf{c}$ & 95 & $93: 7$ \\
$6^{d}$ & $(S)-4 b$ & 92 & $95: 5$ \\
7 & $(S)-6 a$ & 90 & $85: 15$ \\
8 & $(S)-6 b$ & 92 & $94: 6$
\end{tabular}

$\begin{array}{llll}9 & (S)-6 c & 99 & 86: 14 \\ 10 & \text { none }^{e} & 98 & (50: 50)\end{array}$

a Reaction conditions: 1a $(0.10 \mathrm{mmol}), \mathrm{NBP}(0.12 \mathrm{mmol})$, catalyst $(10 \mathrm{~mol} \%, 0.010 \mathrm{mmol})$, toluene $(1.5 \mathrm{~mL})-\mathrm{CH}_{2} \mathrm{Cl}_{2}(0.5$ $\mathrm{mL}),-78{ }^{\circ} \mathrm{C}, 24$ h. ${ }^{b}$ Yield of isolated product $2 \mathrm{a}$. ${ }^{c}$ Determined by HPLC analysis on a chiral stationary phase. ${ }^{d}$ Reaction time $=$ 6 h. ${ }^{e}$ The reaction was performed without a catalyst.

Next, we examined the effects of brominating reagents to gain mechanistic information of the present bromolactonization with bifunctional catalyst (S)-4b (Scheme 2). The enantioselectivity of product $\mathbf{2 a}$ significantly depends on the structure of the brominating reagents. Reactions with brominating reagents possessing 5-membered ring structures gave product $2 \mathrm{a}$ with high levels of enantioselectivity (88:1294:6 er). The reaction with dibromoisocyanuric acid (DBI) possessing a 6-membered ring structure provided $2 \mathrm{a}$ with a moderate level of enantioselectivity (71:29 er). On the other hand, acyclic brominating reagent, such as a $\mathrm{N}$ bromoacetamide (NBA), produced $2 \mathbf{a}$ with a low level of enantioselectivity (66:34 er). Additionally, the reaction with bromine $\left(\mathrm{Br}_{2}\right)$ provided $2 \mathrm{a}$ as a racemate. ${ }^{10}$ These results suggest that the corresponding imide anions generated from brominating reagents are involved in the stereo-determining steps in the present reaction system.

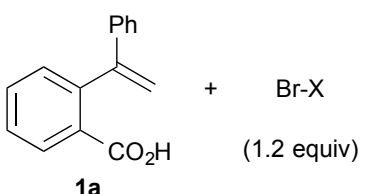

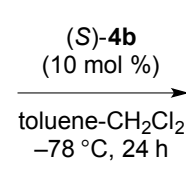

5-membered ring

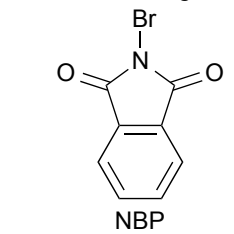

98\%, $94: 6$ er

6-membered ring<smiles>O=c1[nH]c(=O)n(Br)c(=O)n1Br</smiles>

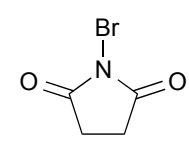

NBS $85 \%, 88: 12$ er acyclic<smiles>CC(=O)NBr</smiles>

NBA

$99 \%, 66: 34$ er

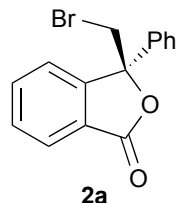

2a (1)
2 Effect of brominating reagents.

The assumed catalytic cycle for the present attempts at bromolactonization using bifunctional sulfide catalyst (S)-4b appear in Scheme 3. In that scheme, when the chiral sulfide catalyst (S)-4b is mixed with NBP, bromosulfonium phthalimide is formed ( $\mathbf{A}$ in Scheme 3 ). The position of the phthalimide anion is fixed by the hydrogen-bonding interaction with a hydroxy group of the catalyst. The alkene portion of substrate $\mathbf{1}$ is then activated by the bromosulfonium moiety to form a cyclic bromonium ion intermediate (B in Scheme 3). Simultaneously, 
a carboxylic acid moiety of $\mathbf{1}$ is also activated by the phthalimide anion, which serves as a Brønsted base, to provide a wellorganized transition-state (TS). Highly enantioselective lactonization then occurs to provide target product $\mathbf{2}$ with the regeneration of catalyst (S)-4b. Based on our previously reported associative TS model involving succinimide anion as the stereo-determining cyclization step, ${ }^{6}$ a plausible TS model for the (S)-4c-catalyzed bromolactonization with $\mathrm{N}$ bromosuccinimide (NBS) was proposed via DFT-computed molecular modeling ( $\mathbf{B}^{\prime}$ in Scheme 3). ${ }^{11}$ The sulfide and naphthol moieties of (S)-4c capably arrange the positions of the bromonium ion generated from carboxylic acid $\mathbf{1}$ and the succinimide anion via sulfide/bromonium interaction and the exhaustive hydrogen bonding network, respectively. The proposed TS model thoroughly explains the crucial role of the naphthol moiety of (S)-4, which was revealed in the control experiments (entries 1-3 in Table 1 ).
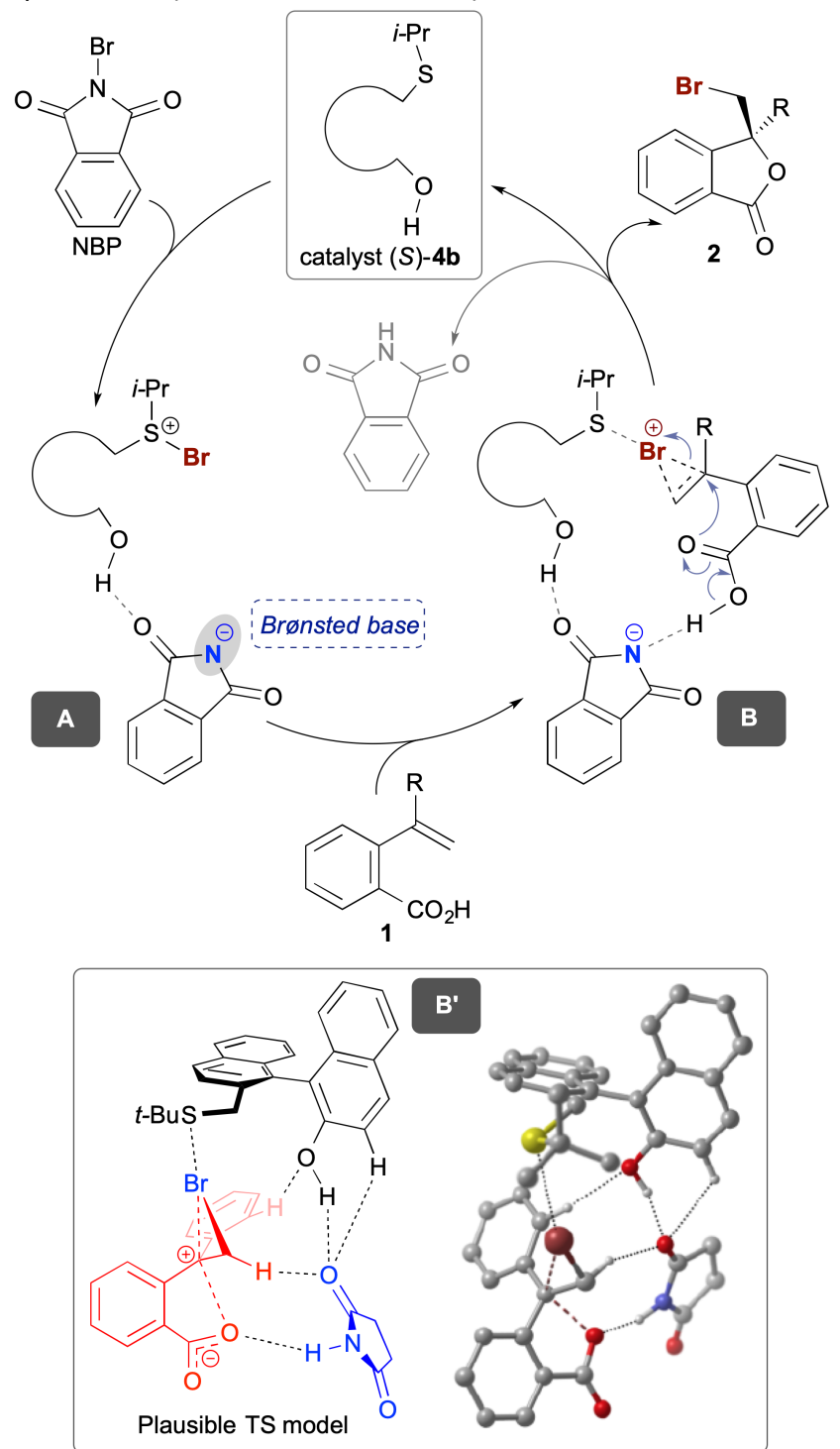

Scheme 3 Proposed catalytic cycle and transition-state structure.

With the effective bifunctional sulfide catalysts (S)-4 in hand, we studied the substrate generality of the bromolactonization of $\mathbf{1}$ to produce various 3,3-disubstituted phthalides $\mathbf{2}$ (Scheme 4). In general, good to high levels of enantioselectivity were observed in the synthesis of 3 -arylphthalides $(\mathbf{2} \mathbf{a}-\mathbf{h})$. Unfortunately, 3-alkylphthalides such as 3-methylphthalide $\mathbf{2 i}$ and $\mathbf{3}$-butylphthalide $\mathbf{2} \mathbf{j}$ were obtained with only low levels of enantioselectivity. 3-Arylphthalides possessing substituents on the aromatic ring at the phthalide core could also be obtained with good to high levels of enantioselectivity $(\mathbf{2 k}-\mathbf{n}) .{ }^{12}$
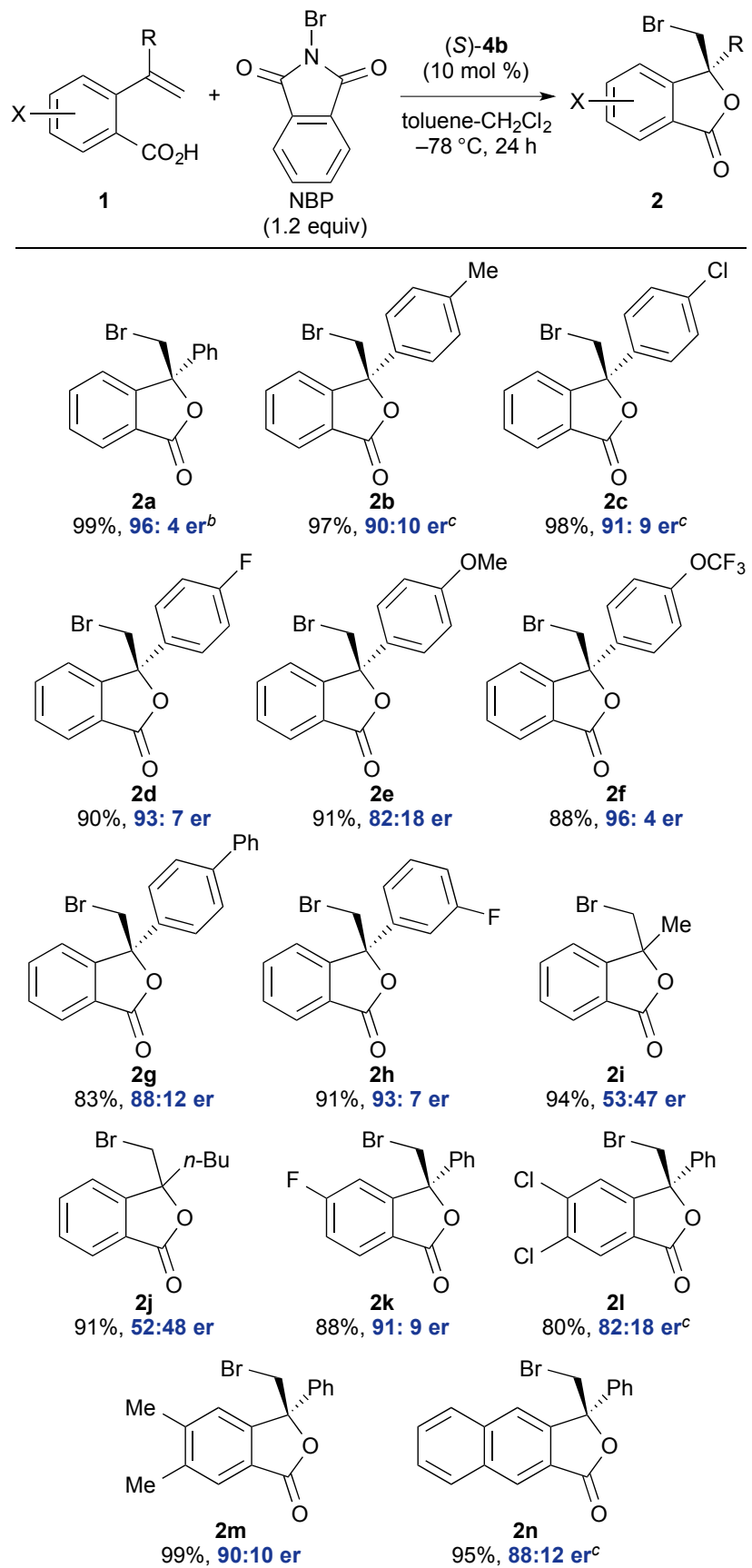

Scheme 4 Scope and limitation. ${ }^{a}$ a Reaction conditions: $1(0.10$ $\mathrm{mmol})$, NBP (0.12 mmol), (S)-4b (10 mol \%, $0.010 \mathrm{mmol})$, toluene $(1.5 \mathrm{~mL})-\mathrm{CH}_{2} \mathrm{Cl}_{2}(0.5 \mathrm{~mL}),-78{ }^{\circ} \mathrm{C}, 24 \mathrm{~h} .{ }^{b}$ The reaction was conducted on a 10 -fold scale (1a: $1.0 \mathrm{mmol}) .{ }^{c}$ The reactions with $\mathbf{1 b}, \mathbf{1 c}, \mathbf{1}$, and $\mathbf{1 n}$ were performed using catalyst $(S)-\mathbf{4 c}$. 
To show additional utility of the present method for the asymmetric synthesis of 3,3-disubstituted phthalides, we examined transformations of the bromo groups in optically active bromolactonization products $\mathbf{2}$ to form other functional groups (Scheme 5). The bromo group of $\mathbf{2 a}$ was removed via reduction with tributyltin hydride under radical conditions to provide 7. Allylation with allyltributyltin was also performed to obtain 8. Introductions of heteroatom substituents were also possible via nucleophilic substitutions, and product $\mathbf{9}$ possessing a thiophenyl group was synthesized via a reaction with thiophenol. It is noteworthy that these reactions proceeded with no loss of enantioselectivity.

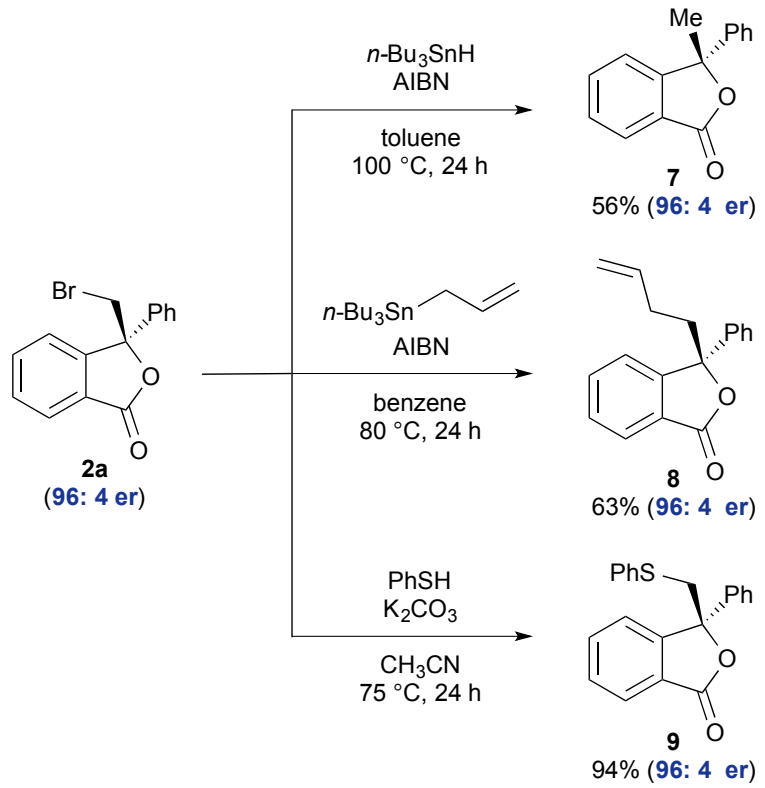

Scheme $\mathbf{5}$ Conversions of product $\mathbf{2 a}$.

\section{Conclusions}

In summary, we have successfully achieved the highly enantioselective synthesis of 3,3-disubstituted phthalides possessing a chiral quaternary carbon center via catalytic asymmetric bromolactonization using BINOL-derived bifunctional sulfide catalysts. The importance of the hydroxy group on the chiral sulfide catalysts to recognize the imide anion generated from brominating reagents was clarified based on the results of control experiments. The great utility of the present synthetic protocol was demonstrated in transformations of the bromo group in optically active phthalide products. Further applications of bifunctional sulfide catalysts to other asymmetric reactions are currently under way by our research group.

\section{Conflicts of interest}

There are no conflicts to declare.

\section{Acknowledgements}

This work was supported by JSPS KAKENHI Grant Numbers JP16K05780 (for S.S.), JP18H04660 (Hybrid Catalysis for M.Y.), the Cooperative Research Program of "Network Joint Research Center for Materials and Devices" (20181268 for S.S.), and Shorai Foundation for Science and Technology (for S.S.).

\section{Notes and references}

1 For reviews on phthalides, see: (a) J. J. Beck and S.-C. Chou, J. Nat. Prod., 2007, 70, 891; (b) R. Karmakar, P. Pahari and D. Mal, Chem. Rev., 2014, 114, 6213.

2 For examples of catalytic asymmetric synthesis of chiral phthalides, see: (a) M. Kitamura, T. Ohkuma, S. Inoue, N. Sayo, H. Kumobayashi, S. Akutagawa, T. Ohta, H. Takaya and R. Noyori, J. Am. Chem. Soc., 1988, 110, 629; (b) K. Everaere, J.L. Scheffler, A. Mortreux and J.-F. Carpentier, Tetrahedron Lett., 2001, 42, 1899; (c) J.-G. Lei, R. Hong, S.-G. Yuan and G.Q. Lin, Synlett 2002, 927; (d) K. Tanaka, G. Nishida, A. Wada and K. Noguchi, Angew. Chem., Int. Ed., 2004, 43, 6510; (e) H.T. Chang, M. Jeganmohan and C.-H. Cheng, Chem. - Eur. J., 2007, 13, 4356; (f) K. Tanaka, T. Osaka, K. Noguchi and M. Hirano, Org. Lett., 2007, 9, 1307; (g) J. Luo, H. Wang, F. Zhong, J. Kwiatkowski, L.-W. Xu and Y. Lu, Chem. Commun., 2012, 48, 4707; (h) F. Zhong, J. Luo, G.-Y. Chen, X. Dou and Y. Lu, J. Am. Chem. Soc., 2012, 134, 10222; (i) J. Luo, C. Jiang, H. Wang, L.W. Xu and Y. Lu, Tetrahedron Lett., 2013, 54, 5261; (j) J. Luo, H. Wang, F. Zhong, J. Kwiatkowski, L.-W. Xu and Y. Lu, Chem. Commun., 2013, 49, 5775; (k) R. Liu, R. Jin, J. An, Q. Zhao, T. Cheng and G. Liu, Chem. - Asian J., 2014, 9, 1388; (I) L. Kong, J. Zhao, T. Cheng, J. Lin and G. Liu, ACS Catal., 2016, 6, 2244; (m) W. Liu, Z.-P. Hu, Y. Yan and W.-W. Liao, Tetrahedron Lett., 2018, 59, 3132; (n) J. M. Cabrera, J. Tauber and M. J. Krische, Angew. Chem., Int. Ed., 2018, 57, 1390.

3 (a) X. Han, C. Dong and H.-B. Zhou, Adv. Synth. Catal., 2014, 356, 1275; (b) D. Parmar, M. S. Maji and M. Rueping, Chem. Eur. J., 2014, 20, 83; (c) H. Egami, J. Asada, K. Sato, D. Hashizume, Y. Kawato and Y. Hamashima, J. Am. Chem. Soc., 2015, 137, 10132.

4 For examples of synthesis of phthalides via intramolecular lactonization of alkenes, see: (a) J. Chen, L. Zhou, C. K. Tan and Y.-Y. Yeung, J. Org. Chem., 2012, 77, 999; (b) S. Hajra, S. M. S. Akhtar and S. M. Aziz, Chem. Commun., 2014, 50, 6913; (c) S. Song, X. Li, X. Sun, Y. Yuan and N. Jiao, Green Chem., 2015, 17, 3285; (d) F. Gelat, M. Coffinet, S. Lebrun, F. AgbossouNiedercorn, C. Michon and E. Deniau, Tetrahedron: Asymmetry, 2016, 27, 980; (e) B. N. Hemric, K. Shen and Q. Wang, J. Am. Chem. Soc., 2016, 138, 5813; (f) E. M. Woerly, S. M. Banik and E. N. Jacobsen, J. Am. Chem. Soc., 2016, 138, 13858; (g) F. Gelat, S. Lebrun, N. Henry, F. AgbossouNiedercorn, C. Michon and E. Deniau, Synlett, 2017, 28, 225.

5 For reviews on catalytic asymmetric halolactonization, see: (a) G. Chen and S. Ma, Angew. Chem., Int. Ed., 2010, 49, 8306; (b) C. K. Tan, L. Zhou and Y.-Y. Yeung, Synlett, 2011, 1335; (c) A. Castellanos and S. P. Fletcher, Chem. - Eur. J., 2011, 17, 5766; (d) S. E. Denmark, W. E. Kuester and M. T. Burk, Angew. Chem., Int. Ed., 2012, 51, 10938; (e) U. Hennecke, Chem. - Asian J., 2012, 7, 456; (f) C. K. Tan and Y.-Y. Yeung, Chem. Commun., 2013, 49, 7985; (g) K. Murai and H. Fujioka, Heterocycles, 2013, 87, 763; (h) C. K. Tan, W. Z. Yu and Y.-Y. Yeung, Chirality, 2014, 26, 328; (i) S. Zheng, C. M. Schienebeck, W. Zhang, H.-Y. Wang and W. Tang, Asian J. Org. Chem., 2014, 3, 366; (j) Y. A. Cheng, W. Z. Yu and Y.-Y. Yeung, Org. Biomol. Chem., 2014, 12, 2333; (k) C. B. Tripathi and S. Mukherjee, Synlett, 2014, 25, 163; (I) A. Sakakura and K. Ishihara, Chem. Rec., 2015, 15, 728; (m) M. H. Gieuw, Z. Ke and Y.-Y. Yeung, Chem. Rec., 2017, 17, 287; (n) Y. Kawato and Y. Hamashima, Synlett, 2018, 29, 1257. 
6 R. Nishiyori, A. Tsuchihashi, A. Mochizuki, K. Kaneko, M. Yamanaka and S. Shirakawa, Chem. - Eur. J., 2018, 24, 16747.

7 For reviews on chiral sulfide catalysts, see: (a) V. K. Aggarwal, Synlett, 1998, 329; (b) V. K. Aggarwal and C. L. Winn, Acc. Chem. Res., 2004, 37, 611; (c) E. M. McGarrigle, E. L. Myers, O. Illa, M. A. Shaw, S. L. Riches and V. K. Aggarwal, Chem. Rev., 2007, 107, 5841; (d) R. Gómez Arrayás and J. C. Carretero, Chem. Commun., 2011, 47, 2207; (e) J. Luo, X. Liu and X. Zhao, Synlett, 2017, 28, 397.

8 For recent examples of chiral sulfide catalysts, see: (a) Z. Ke, C. K. Tan, F. Chen and Y.-Y. Yeung, J. Am. Chem. Soc., 2014, 136, 5627; (b) Z. Ke, C. K. Tan, Y. Liu, K. G. Z. Lee and Y.-Y. Yeung, Tetrahedron, 2016, 72, 2683; (c) X. Liu, R. An, X. Zhang, J. Luo and X. Zhao, Angew. Chem., Int. Ed., 2016, 55, 5846; (d) Q.-Z. Li, X. Zhang, R. Zeng, Q.-S. Dai, Y. Liu, X.-D. Shen, H.-J. Leng, K.-C. Yang and J.-L. Li, Org. Lett., 2018, 20, 3700; (e) Q. Cao, J. Luo and X. Zhao, Angew. Chem., Int. Ed., 2019, 58, 1315.

9 For optimization of the reaction solvents, see Table S1 in Supplementary Information.

10 The reaction with bromine $\left(\mathrm{Br}_{2}\right)$ may proceed via noncatalyzed reaction pathway (background reaction pathway).

11 The transition structure was partially optimized (the reaction center was frozen) at the B3LYP/6-31LAN (LANL2DZ for Br and 6-31G* for the rest) level. For detail, see Supplementary Information.

12 See also, Scheme S1 in Supplementary Information. 